\title{
Atividade farmacológica da monocrotalina isolada de plantas do gênero Crotalaria
}

\author{
José E. R. Honório Júnior, ${ }^{1}$ Paula M. Soares, ${ }^{1,2}$ Célio L. de Melo, ${ }^{1}$ Antônio C. V. Arruda Filho, ${ }^{1}$ \\ José G. Sena Filho, ${ }^{3}$ José M. Barbosa Filho, ${ }^{1,3}$ Francisca Cléa Florenço Sousa, ${ }^{1}$ Marta Maria \\ França Fonteles, ${ }^{1}$ Luzia Kalyne de Almeida Leal, ${ }^{1}$ Maria Goretti Rodrigues de Queiroz, ${ }^{4}$ \\ Silvânia M.M. Vasconcêlos ${ }^{* 1}$
}

${ }^{1}$ Departamento de Fisiologia e Farmacologia, Universidade Federal do Ceará. Rua Cel Nunes de Melo, 1127, Rodolfo Teófilo, 60430-270 Fortaleza-CE, Brasil,

${ }^{2}$ Curso de Educação Física, Universidade Estadual do Ceará. Av. Parajana, 1700, Itapery, 60730-180

Fortaleza-CE, Brasil,

${ }^{3}$ Laboratório de Tecnologia Farmacêutica "Delby Fernandes de Medeiros", Universidade Federal da Paraíba, Caixa Postal 5009, 58051-970 João Pessoa-PB, Brasil,

${ }^{4}$ Departamento de Bioquímica, Universidade Federal do Ceará. Rua Cel Nunes de Melo, 1127, Rodolfo Teófilo, 60430-270 Fortaleza-CE, Brasil.

\begin{abstract}
RESUMO: Crotalaria retusa é uma planta encontrada no Nordeste brasileiro, pertence ao gênero Crotalaria e à família Leguminosae, e possuem mais de seissentas espécies no mundo e mais de quarenta no Brasil. As variedades tóxicas mais conhecidas são C. spectabilis, C. crispata, C. retusa, C. dura e C. globifera. Plantas do gênero Crotalaria são de interesse porque são usadas na medicina popular. Esses gêneros são ricos em alcaloides pirrolizidínicos (AP), que são as principais toxinas e apresentam efeitos pneumotóxicos, nefrotóxicos, cardiotóxicos, fetotóxicos, carcinogênicos, inflamação, hemorragia e fibrose. A monocrotalina é o principal alcaloide pirrolizidínico encontrado nessas plantas e é ativamente oxidada in vivo pelo citocromo $\mathrm{P} 450$ no fígado, formando intermediários altamente reativos tipo pirrólicos que são responsáveis pela ligação cruzada do DNA-DNA e DNAproteína. O presente trabalho teve como objetivo fazer um levantamento bibliográfico via internet, utilizando bancos de dados, programas de pesquisa científica e pesquisa em livros relacionados, acerca da atividade farmacológica e do mecanismo de ação da monocrotalina extraída de plantas do gênero Crotalaria, ressaltando desde os aspectos botânicos da planta, estrutura química dos alcaloides pirrolizidínicos, exemplos experimentais de toxicidade e provável mecanismo de ação.
\end{abstract}

Unitermos: Monocrotalia, hepatotóxico, Crotalaria, dehidromonocrotalina.

ABSTRACT: "Pharmacological activity of monocrotalina isolated from plants of the genus Crotalaria." Crotalia retusa is a plant found in Brazilian Northeast and belongs to the genus Crotalaria and the family Leguminosae, which comprises more than 600 species throughout the world and more than forty in Brazil. The most known toxic species are C. spectabilis, C. crispata, C. retusa, C. dura and C. globifera. Plants of the Crotalaria genus are of great interest because they are used by humans for folk medicine. These plants are rich in pyrrolizidine alkaloids (PA), which are the main toxins that cause effects such as pneumotoxic, nefrotoxic, cardiotoxic, fetotoxic, carcinogenic, inflammation, hemorrhage and fibrosis. Monocrotaline is the main pirrolizidinic alkaloid found in plants and is actively oxidated in vivo by the cytochrome P450 in the liver, yielding highly reactive pyrrolic type intermediates, which are responsible for DNA-DNA and DNA-protein cross-links reaction. The aim of this work is to make a bibliographic survey via internet, using databases, scientific research programs and related books, about pharmacological activity and mechanism of action of monocrotaline extracted from plants of Crotalaria genus, emphasizing plant botanical aspects, chemical structure of pirrolizidinic alkaloid, experimental examples of toxicity and probable action mechanism.

Keywords: Monocrotaline; hepatotoxity; Crotalaria; dehydromonocrotaline. 


\section{INTRODUÇÃO}

Plantas do gênero Crotalaria pertencem à família Leguminosae com mais de seissentas espécies e crescem abundantemente em zonas tropicais e subtropicais sendo numerosas na África, Índia, México e Brasil que representam os principais centros de diversidade das espécies (Cheecke, 1988; Palomino \& Vasquez, 1991). Por serem plantas invasoras, as Crotalárias são facilmente encontradas em plantações de grãos e em pastagens. Na região Neotropical, aproximadamente setenta espécies ocorrem desde o sul dos Estados Unidos até a Argentina subtropical e Uruguai. As espécies pertencentes ao gênero Crotalaria são plantas herbáceas com cerca de $50 \mathrm{~cm}$ de altitude ou arbustos com até $3 \mathrm{~m}$, com folhas digitado-trifolioladas, unifolioladas ou simples; flores predominantemente amarelas; androceu formando um tubo monadelfo aberto na base com anteras dimorfas e legumes inflados. As espécies do gênero possuem considerável plasticidade, adaptando-se às diferentes condições ambientais. Podem ocorrer em variados tipos de habitats, como áreas próximas de rios, morros litorâneos, restingas, orla de matas, campos e cerrados. As espécies são oportunistas, muito comuns em locais alterados como margem de estradas e como invasoras de culturas (Flores \& Miotto, 2005). As plantas do gênero Crotalaria, no Brasil, recebem comumente nomes como "xique-xique", "guizo-de-cascavel", "chocalho-de-cascavel" e possuem vagens secas que quando tocadas se assemelham ao som emitido pela cauda da cascavel (Williams \& Molyneux, 1987).

No Brasil, já foram encontradas aproximadamente mais de quarenta espécies, sendo muitas consumidas pelos animais, principalmente nos períodos de escassez de alimentos (Hoehne, 1939; Tokarnia et al., 2000). As variedades tóxicas mais conhecidas são Crotalaria spectabilis, Crotalaria crispata, Crotalaria retusa, Crotalaria dura e Crotalaria globifera (Barri \& Adam, 1981).

O uso principal destas plantas se dá na agricultura, pela adubação verde, cobertura vegetal do solo auxiliando o plantio direto. Plantas do gênero Crotalaria também são de grande interesse devido às perdas econômicas causadas por intoxicação do gado e devido à exposição da população humana que usam muitas dessas plantas na medicina popular (Atal \& Sawhney, 1973; Mattocks, 1986). Trabalhos mostram que a Crotalaria apresenta atividade depressora no sistema nervoso central na dose de 50 e $100 \mathrm{mg}$ em camundogos (Honório Júnior et al., 2008).

Com o propósito de estudar as ações tóxicas e farmacológicas da monocrotalina extraída do Gênero Crotalaria foi realizado extensa revisão bibliográfica pela internet de textos e artigos relacionados ao tema. Os programas de bancos de dados utilizados foram a Pubmed e Lilacs.

\section{ALCALOIDES PIRROLIZIDÍNICOS}

Essas plantas são ricas em alcaloides pirrolizidínicos (AP) que são as principais toxinas derivadas de plantas que acometem humanos e animais (Mattocks, 1986; Huxtable, 1990).

Em diversos países foram descritos casos de intoxicação por Crotalaria, contendo alcaloides pirrolizidínicos (AP), em equinos (Gibbons et al., 1953, Gardiner et al., 1965; Arzt \& Mount, 1999; Nobre et al., 2004a, b), bovinos (Barri \& Adam, 1981; Winter et al., 1990), suínos (Peckham et al., 1974; Mcgrath et al., 1975), aves (Norton \& O'rourke, 1979; Alfonso et al., 1993) e caprinos (Nobre et al., 2005, Barri \& Adam, 1984). C. retusa e C. crispata são responsáveis pela doença denominada Kimberley horse disease ou Walkabout disease na Austrália (Rose et al., 1957a, b; Gardiner et al., 1965).

A monocrotalina (1) é o principal AP encontrado nas plantas do gênero Crotalaria e, embora seja um alcaloide primariamente hepatotóxico, efeitos pneumotóxicos, nefrotóxicos, cardiotóxicos, teratogênicos e carcinogênicos também estão relacionados a ele (Mattocks, 1986; Thomas et al., 1996; Ribeiro et al., 1993; Cheecke, 1998; Medeiros et al., 2000; Kosogof et al., 2001).

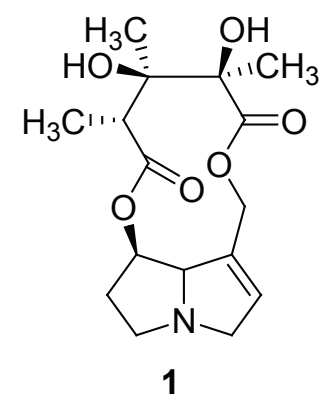

Nos pulmões, as principais alterações observadas são edema e congestão pulmonar com áreas consolidadas no parênquima, provocando lesão intersticial e arteriolar pulmonar, inflamação, hemorragia e fibrose (Gardiner et al., 1965; Newberne et al., 1974; Nobre et al., 1994; Baybutt \& Molteni, 1999; Baybutt et al., 2002; Copple et al., 2002).

Schraufnagel (1990) estudando pulmões de ratos tratados com monocrotalina (MCT) observou a formação de novos vasos. A descoberta da angiogênese na superfície pleural e na árvore broncovascular, mas não nos capilares alveolares, sugerindo uma diferenciação básica de como esses capilares respondem aos estímulos angiogênicos.

Nos rins, podem ocorrer lesões renais que podem levar à morte (Jubb et al., 1993). As principais alterações observadas são danos tubulares e glomerulonefrite (Hayashi \& Lalich, 1967; Carstens \& Allen, 1970; McGrath et al., 1975; Figueredo et al., 1987).

Ocorre gigantismo citoplasmático e nuclear (megalocitose) em células hepáticas (Bull, 1955; Cheeke \& 
Shull, 1985; Mattocks, 1986; Thomson, 1990; Jubb et al., 1993; Gibbons et al., 1953; Gardiner et al., 1965; Arzt \& Mount, 1999), necrose (Gardiner et al., 1965), vacuolização de hepatócitos (Nobre et al., 1994), hemorragia (Arzt \& Mount, 1999). Outras lesões incluem fibrose progressiva, proliferação de ductos biliares (Cheeke \& Shull, 1985; Mattocks, 1986; Thomson, 1990) e veno-oclusão em regiões hepáticas (Cheeke Shull, 1985; Mattocks, 1986). Há diminuição das funções metabólicas hepáticas; os níveis de proteína sérica tornam-se diminuídos, devido à redução na síntese protéica (Miranda et al., 1980), levando a ascite e edema (Cheeke \& Garman, 1974).

\section{ESTRUTURA QUÍMICA DOS ALCALOIDES PIRROLIZIDÍNICOS}

Os alcaloides são compostos que contem nitrogênio em um anel heterocíclico e são geralmente de caráter básicos (tipo alcalino=alcaloide). Eles são de sabor amargo, fisiologicamente e farmacologicamente ativos e funcionam como uma defesa química das plantas contras herbívoros. Os AP constituem um grande grupo de alcaloides contendo um núcleo pirrolizidino sendo amplamente disseminados, tanto geograficamente quanto botanicamente. Muitos desses AP são hepatotóxicos, causando danos irreversíveis ao fígado e outros são carcinogênicos (McLean, 1970; Cheecke \& Shull, 1985; Prakash et al., 1999; Cheeke, 1988).

Segundo Mattocks (1986), a hepatotoxidade dos APs está associada à insaturação entre os carbonos 1 e 2 que, na presença de oxidases, leva a formação de pirróis. Estes se associam a grupos nucleofílicos de macromoléculas, como sulfidrila, hidroxila e grupos amino de enzimas, globulinas, hemoglobinas, bem como purinas e pirimidinas, formando ligações cruzadas irreversíveis com o DNA e RNA, e acarretando em efeitos citotóxicos, mutagênicos e carcinogênicos (Nobre et al., 2004a).

\section{TOXICIDADE: DADOS EXPERIMENTAIS}

Segundo Peckham et al. (1974), em um rebanho de suínos ao qual foi fornecida uma ração contendo milho contaminado com $0,5 \%$ de sementes de C. spectabilis, morreram 76 de um total de 150 animais. As primeiras mortes ocorreram seis semanas após o início do consumo da ração e três semanas após a queda de pêlos do corpo dos animais, sendo que esse foi o primeiro sinal clínico observado. Outros sinais clínicos foram anemia, melena, emaciação e redução de peso. Foram colhidas amostras de sangue de dezesseis animais, dos quais seis apresentavam sintomas da intoxicação e dez apresentavam-se aparentemente sadios. Destes dezesseis animais, um apresentou leucopenia e severa anemia; outro animal apresentou leucocitose; três suínos estavam levemente anêmicos e onze animais exibiam hemogramas normais. Com o soro dos dez animais aparentemente sadios foram realizadas as seguintes provas bioquímicas: uréia nitrogenada sanguínea, fosfatase alcalina, transaminase glutâmica oxalacética-TGO (AST) e transaminase glutâmica pirúvica-TGP (ALT). Foi encontrado um pequeno aumento na atividade média da fosfatase alcalina, TGO (AST) e TGP (ALT), quando comparada à atividade dessas enzimas no soro de suínos que não receberam ração contaminada com sementes de C. spectabilis. Após o consumo da ração ocorreram mortes durante as dezesseis semanas de observação, entretanto a maioria destas ocorreu entre a $8^{\mathrm{a}}$ e $12^{\mathrm{a}}$ semanas. Os efeitos tóxicos das sementes foram evidenciados cinco meses após a remoção destas da dieta. Os principais achados patológicos foram úlceras gástricas, hemoperitônio, atrofia e fibrose hepáticas, fibrose renal com cistos glomerulares e pneumonia proliferativa intersticial. Lesões microscópicas de maior valor diagnóstico foram hipertrofia de hepatócitos e de células epiteliais dos túbulos contorcidos renais, com cariomegalia. A severidade dos efeitos causados pela intoxicação por Crotalaria foi grandemente influenciada pela idade dos suínos. Leitões desmamados um pouco antes de receberem ração contaminada foram mais severamente afetados e apresentaram taxa de mortalidade próxima a $80 \%$, enquanto que suínos com peso próximo ao do abate $(100 \mathrm{~kg})$ tiveram uma mortalidade de $8 \%$ e não houve morte entre as fêmeas (Peckham et al., 1974).

Equinos intoxicados por Crotalaria retusa apresentam um quadro clínico de anorexia, atordoamento, mal estado geral, irritabilidade, bocejos, espasmos musculares, incoordenação, cabeça baixa, agressividade, andar a esmo e galope sem rumo (Gardiner et al., 1965; Nobre et al., 2004a). Sintomatologia semelhante foi relatada na intoxicação de eqüinos por outras espécies de Crotalaria (Arzt \& Mount, 1999; Nobre et al., 2004 a, b). Os achados de necropsia são característicos de uma doença hepática crônica. O fígado encontra-se firme, aumentado de volume (Gibbons et al., 1953; Gardiner et al., 1965; Nobre et al., 1994; Arzt \& Mount, 1999) e com aspecto mosqueado (Arzt \& Mount, 1999). Nos pulmões, as principais alterações observadas são edema e congestão pulmonar com áreas consolidadas no parênquima (Gardiner et al., 1965; Nobre et al., 1994). Os pulmões podem apresentar alveolite fibrosante difusa, com espessamento de septos interalveolares, edema e infiltrado inflamatório mononuclear e, principalmente, macrófagos espumosos (Gardiner et al., 1965; Nobre et al., 1994). Equinos submetidos a estresse metabólico ou nutricional na seca, carga de trabalho pesada ou gravidez, são mais prováveis de serem afetados pela doença (Curran et al., 1996). A doença crônica é a forma usual da intoxicação e os sinais clínicos ocorrem semanas a meses após a planta ter sido ingerida. Os danos hepáticos são progressivos e a morte pode ocorrer meses ou até mesmo anos após a ingestão de plantas contendo AP (Cheeke, 1998).

Nobre et al. (2005) trabalhando com um rebanho de oitenta ovelhas mostraram alguns sinais de intoxicações agudas como anorexia, severa depressão, icterícia e 
diminuição da atividade locomotora. Lesões histológicas do fígado foram caracterizadas com necrose centrilobular. Alguns animais foram tratados dando sementes de $C$. retusa nas doses de 2,5 (duas ovelhas); $5 ; 10 ; 20$ e $40 \mathrm{~g} /$ $\mathrm{kg}$ (uma ovelha para cada dose) por ingestão. Os sinais clínicos e graves foram os mesmo observados nos rebanhos de ovelhas no campo. Nobre et al. (2004b) observaram os mesmos sinais clínicos em equinos e asininos que se intoxicaram ingerindo as sementes durante a alimentação. Além disso, a necropsia revelou astrócitos Alzheimer tipo II, isolados ou em grupos principalmente no núcleo caudato e córtex.

Barreto et al. (2006) demonstraram resultados que indicam uma ação direta da MCT em culturas celulares de astrócitos corticais de ratos, alterando a morfologia e o crescimento celular, e sugerem que a resposta astrocitária a este alcalóide pode estar relacionada aos danos no SNC observados em animais intoxicados. Baixas concentrações de dehidromonocrotalina mostraram uma reação astrogliolítica e em altas concentrações (100-500 $\mu \mathrm{M})$ os astrócitos apresentaram diminuição do corpo celular, fraca expressão da proteína GFAP (glial fibrilary acidic protein) ligada às cromatinas nucleares, retração dos seus dendritos, condensação e fragmentação da cromatina em uma importante proporção (30\%) sugerindo sinais de apoptose (Barreto et al., 2008). Sinais de apoptose também foram vistos, tanto em baixa com em altas concentrações de monocrotalina, nas células endoteliais das artérias pulmonares (Thomas et al., 1998).

O mecanismo exato da toxicidade causada pela MCT ainda não está determinado, mas sabe-se que é necessária a sua biotransformação pelo fígado no metabólito reativo dehidromonocrotalina (DHM), o qual interfere na síntese de DNA e proteínas (Petry et al., 1984; Butler et al., 1970).

\section{PROVÁVEL MECANISMO DE AÇ̃̃O}

Esses alcaloides pirrolizidínicos são ativamente oxidados in vivo formando intermediários altamente reativos tipo pirrólicos que são responsáveis pela ligação cruzada com o DNA(Kosogof et al., 2001; Tepe \& Williams, 1999). Assim, a monocrotalina requer a bio-ativação em dehidromonocrotalina pelo citocromo P450 no fígado, uma reação bifuncional de um agente alcalínico que se liga ao DNA celular e protéico in vivo. Dehidromonocrotalina parece ser neutralizada pela conjugação com a glutationa (GSH). Assim, aminoácido tipo cisteína contendo sulfil e taurina previne contra o efeito tóxico da monocrotalina (Yan \& Huxtable, 1996).

A oxidação do substrato ligado à energia reduz produtos equivalentes, que são transferidos via nucleotídeos piridina (NADH) ou flavina $\left(\mathrm{FADH}_{2}\right)$ na cadeia respiratória. Um gradiente eletroquímico de prótons tem o potencial membrânico como principal componente, capaz de sustentar a síntese de ATP em que foi gerado por ele (Nicholls, 1976).

Tepe\&Williams(1999)descreveramasemi-síntese e a ligação cruzada com o DNA dos primeiros precursores engatilhados fotoquimicamente de dehidromonocrotalina. Algumas substâncias como antibiótico e antitumorais que ocorrem naturalmente são ativados oxidativamente ou redutivamente in vivo formando um intermediário tipo pirroli altamente reativo que são responsáveis por uma reação de ligação cruzada com o DNA. Essa via oxidativa ou redutiva pode leva a uma variedade de efeitos tóxicos.

Precursor engatilhado fotoquimicamente de um alcaloide pirrolizidínico que gerava dehidromonocrotalina sobre ativação fotoquímica. Essa oxidação resulta da ativação eletrofílica da posição C-7 e C-9 da molécula de pirrole, via conjugação com um par único de nitrogênio pirrole que é propenso a ataque nucleofílico pelo DNA. A especificidade da ligação cruzada com o DNA dessa reação ocorre no lugar ${ }^{5} \mathrm{CpG}^{3}$ ' (no par de nucleotídeos Citocina e Guanina) via amina exocíclica do resíduo dG na curva menor (Kosogof et al., 2001; Tepe \& Williams, 1999).

Wallace \& Starkov (2000) recordam que essa conexão com as mitocôndrias têm freqüentemente sido consideradas um importante alvo de xenobióticos que podem causar injúria celular via depleção do ATP.

Koosogof et al. (2001) mostraram que a viabilidade estruturalmente escondida de alguns precursores pirolizidinos reativos ao DNA podem serem planejados e sintetizados a partir de modificações de oligonucleotídeos específicos. Tais agentes asseguram promessas de ferramentas úteis para entender o mecanismo de ligação cruzada de DNA-DNA e DNA-proteína.

O complexo I fornece o lugar da entrada dos elétrons dos NADH para a cadeia transportadora de elétrons mitocondrial. O complexo I dos mamíferos é uma enzima que contém 46 diferentes subunidades com uma massa molecular de $980 \mathrm{kDa}$. Essa organização supramolecular e a relação estrutural restante da cadeia respiratória, contudo, ainda permanecem desconhecidas. A transferência de elétrons pelo NADH (oxidação) via complexo I reduz a ubiquinona (coenzima Q) gerando parte do gradiente eletroquímico requerido pela síntese de ATP. Contudo, a inibição do complexo I da cadeia respiratória pode constituir um importante mecanismo de deleção do ATP da célula e assim levar a morte celular (Lenaz et al., 2006; Caroll et al., 2003).

\section{CONSIDERAÇÕES FINAIS}

Apesar de seu uso se fazer presente na agricultura, existe a necessidade de aprimorar os estudos de plantas do gênero Crotalaria, voltado principalmente para seu principal constituinte tóxico (monocrotalina). Novas informações são necessárias, no sentido de aumentar as o conhecimento dos seus efeitos no organismo de humanos e animais e desvendar seu exato mecanismo de ação, visando descobrir drogas capazes de intervir em sua via de ação, protegendo ou revertendo seus efeitos nocivos. 


\section{REFERÊNCIAS}

Alfonso HA, Sanchez LM, Figueredo MA, Gómez BC 1993. Intoxication due to Crotalaria retusa and C. spectabilis in chickens and geese. Vet Hum Toxicol 35: 539-544.

Arzt J, Mount M 1999. Hepatotoxicity associated with pyrrolizidine alkaloid (Crotalaria spp) ingestion in a horse on Easter Island. Vet Hum Toxicol 41: 96-99.

Atal CK, Sawhney RS 1973. The pyrrolizidine alkaloids from Indian Crotalarias. Indian J Pharm 35: 1-12.

Barreto RA, Hughes JB, Souza CS, Silva VDA, Silva AR, Velozo ES, Batatinha MJM, Costa MFD, El-bacha RS, Costa SL 2006. O alcaloide monocrotalina, extraído de Crotalaria retusa, altera a expressão GFAP, a morfologia e o crescimento de culturas primárias de astrócitos. Rev Bras Saude Prod An 7: 112-127.

Barreto RA, Sousa CS, Silva VDA, Silva AR, Velozo ES, Cunha SD, Costa MFD, El-Bacha RS, Costa SL 2008. Monocrotaline pyrrol is cytotoxic and alters the patterns of GFAP expression on astrocyte primary cultures. Toxicol in Vitro 22: 1191-1197.

Barri MES, Adam SEI 1984. Effects of Crotalaria saltiana on Nubian Goats. Vet Hum Toxicol 26: 476-480.

Barri MES, Adam SEI 1981. The toxicity of Crotalaria saltiana to calves. J Comp Pathol 91: 621-627.

Baybutt RC, Molteni A 1999. Dietary X-carotene protects lung and liver parenchyma of rats treated with monocrotaline. Toxicology 137: 69-80.

Baybutt RC, Rosales C, Brady H, Molteni A 2002. Dietary fish oil protects against lung and liver inflammation and fibrosis in monocrotaline treated rats. Toxicology 175: 1-13.

Bull LB 1955. The histological evidence of liver damage from pyrrolizidine alkaloids: megalocytosis of the liver cells and inclusion globules. Aust Vet J 18: 33-40.

Butler WH, Mattocks AR, Barnes JM 1970. Lesions in the liver and lungs of rats given pyrrole derivatives of pyrrolizidine alkaloids. J Pathol 100: 169-175.

Caroll J, Fearnley IM, Shannon RJ, Hirst J, Walker JE 2003. Analysis of the subunit composition of complex I from bovine heart mitochondria. Mol Cell Proteom 2: 117126.

Carstens LA, Allen JR 1970. Arterial degeneration and glomerular hyalinization in the kidney of monocrotaline-intoxicated rats. Am J Pathol 60: 75-90.

Cheecke PR 1998. Natural toxicants in feeds, forages and poisonous plants. Danville: Interstate Publishers.

Cheeke PR, Garman GR 1974. Influence of dietary protein and sulfur amino acid levels on the toxicity of Senecio jacobaea (Tansy Ragwort) to rats. Nutr Rep Int 9: 197202.

Cheeke PR, Shull LR 1985. Natural toxicants in feeds and poisonous plants. Westport: AVI Publishing Company.

Cheeke PR 1988. Toxicity and metabolism of pyrrolizidine alkaloids. J Anim Sci 66: 2343-2350

Copple BL, Banes A, Ganey PE, Roth RA 2002. Endothelial cell injury and fibrin deposition in rat liver after monocrotaline exposure. Toxicol Sci 65: 309-318.

Curran JM, Sutherland RJ, Peet RL 1996. A screening test for subclinical liver disease in horses affected by pyrrolizidine alkaloid toxicosis. Aust Vet J 74: 236-240.

Figueredo MLA, Rodríguez J, Alfonso HA 1987. Patologia de la intoxicacion experimental aguda por Crotalaria retusa y C. spectabilis em pollos. Rev Cubana Cienc Vet 18: 63-71.

Flores AS, Miotto STS 2005. Aspectos fitogeográficos das espécies de Crotalaria L. (Leguminosae-Faboideae) na Região Sul do Brasil. Acta Bot Bras 19: 245-249.

Gardiner MR, Royce R, Bokor A 1965. Studies on Crotalaria crispata, a newly recognised cause of Kimberley horse disease. J Comp Pathol 89: 43-53.

Gibbons WJ, Durr EH, Cox SA 1953. An outbreak of cirrosis of the liver in horses. N Am Vet 34: 556-558.

Hayashi Y, Lalich JJ 1967. Renal and pulmonary alterations induced in rats by a single injection of monocrotaline. $P$ Soc Exp Biol Med 124: 392-396.

Hoehne FC 1939. Leguminosas papilionaceas: Crotalaria e Lupinus. In: Hoehne, F.C.. (ed.). Plantas e substâncias vegetais tóxicas e medicinais. São Paulo: Graphicars,. p. 137-196.

Honório Júnior JER, Chaves EMC, Silva, MCC, Martins ALAR, Escudeiro SS, Filho JGS, Queiroz MGR, Filho JMB, Vasconcelos SMM 2008. Efeitos centrais da monocrotalina, extraída da Crotalaria retrusa em camundongos. In: III Reunião Regional FeSBE. Fortaleza, Brasil.

Huxtable RJ 1990. Activation and pulmonary toxicity of pirrolizidine alkaloids. Pharmacol Therapeut 47: 371389.

Jubb KVF, Kennedy PC, Palmer N 1993. Pathology of domestic animals. New York: Academic Press.

Lenaz G, Fatoa R, Genovaa ML, Bergaminia C, Bianchia C, Biondia A 2006. Mitochondrial complex I: structural and functional aspects. Biochim Biophys Acta 1757: 14061420 .

Kosogof C, Tepe JJ, Williams, RM 2001. DNA cross-linking by a phototriggered pyrrolic progenitor developed from monocrotaline. Tetrahedron Lett 42: 6641-6643.

Mattocks AR 1986. Toxicology of pyrrolizidine alkaloids in animal. In: Mattocks A R . Chemistry and toxicology of pyrrolizidine alkaloids. New York: Academic Press, p. 191-219.

Mcgrath JPM, Duncan JR, Munnell JF 1975. Crotalaria spectabilis toxicity in swine: characterization of the renal glomerular lesion. J Comp Pathol 85: 185-194.

Mclean EK 1970. The toxic actions of pyrrolizidine (Senecio) alkaloids. Pharm Vet 22: 429-483.

Medeiros RMT, Górniak SL, Guerra JL 2000. Fetotoxicity and reproductive effects of monocrotaline in pregnant rats. $J$ Ethnopharmacol 69: 181-188.

Miranda CL, Cheeke PR, Schmitz JA, Buhler DR 1980. Toxicity of Senecio jacobaea (Tansy Ragwort) in rats. Toxicol Appl Pharm 56: 432-442. 
Newberne PM, Chan WCM, Rogers AE 1974. Influence of light, riboflavin, and carotene on the response of rats to the acute toxicity of aflatoxin and monocrotaline. Toxicol Appl Pharm 28: 200-208.

Nicholls DG 1976. The bioenergetics of brown adipose tissue mitochondria. FEBS Lett. 61: 103-110.

Nobre D, Dagli MLZ, Haraguchi M 1994. Crotalaria juncea intoxication in horses. Vet Hum Toxicol 36: 445-448.

Nobre VMT, Riet-Correa F, Dantas AFM, Tabosa IM, Medeiros RMT, Barbosa Filho JM 2004a. Intoxication by Crotalaria retusa in ruminants and eqüidae in the state of Paraíba, northeastern Brazil. In: Acamovich T., Stewart C.S. \& Pennycott T.W. (ed.). Plant Poisoning and related Toxins. CAB International, Glasgow, UK, p. 275-278.

Nobre VMT, Riet-Correa F, Barbosa Filho JM, Dantas AFM, Tabosa IM, Vasconcelos JS 2004b. Intoxicação por Crotalaria retusa (Fabaceae) em Equíneos no semi-árido da Paraíba. Pesq Vet Bras 24: 132-143.

Nobre VMT, Dantas AFM, Riet-Correa F, Barbosa Filho JM, Tabosa IM, Vasconcelos JS 2005. Acute intoxication by Crotalaria retusa in sheep. Toxicon 45: 347-352.

Norton JH, O'rourke PK 1979. Toxicity of Crotalaria goreensis for chickens. Aust Vet J 55: 173-174.

Palomino G, Vázquez R, 1991. Cytogenetic studies in mexican populations of species of Crotalaria (Leguminosae Papilionoideae). Cytologia 56: 343-351.

Peckham JC, Sangster LT, Jones JROH 1974. Crotalaria spectabilis poisoning in swine. $J$ Am Vet Med Assoc 165: 633-638.

Petry TW, Bowden G T, Huxtable RJ, Sipes IG 1984. Characterization of hepatic DNA damage induced in rats by the pyrrolizidine alkaloid monocrotaline. Cancer Res 44: 1505-1509.

Prakash AS, Pereira TN, Reilly PE, Seawright AA 1999. Pyrrolizidine alkaloids in human diet. Mutat Res 5: 5367.

Ribeiro LR, Silva AR, Bautista AR, Costa SL, Sales LA, Rios AC, Salvadori DM 1993. Clastogenic effect of extracts obtained from Crotalaria retusa L. and Crotalaria mucronata Desv. on mouse bone marrow cells. Mutat Res 300: 253-258.

Rose AL, Gardner C A, McConnell JD, Bull LB 1957a. Field and experimental investigation of "walk about" disease of horses (Kimberley horse disease) in Northern Australia: Crotalaria poisoning in horses. Aust Vet J 33: 25-33.

Rose AL, Gardner CA, McConnell JD, Bull LB 1957b. Field and experimental investigation of "walk about" disease of horses (Kimberley horse disease) in Northern Australia: Crotalaria poisoning in horses. Aust Vet J 34: 49-62.

Schraufnagel DE 1990. Monocrotaline-induced angiogenesis. Am J Pathol 137: 1083-1090.

Tepe JJ, Williams RM 1999. DNA cross-linking by a phototriggered dehydromonocrotaline progenitor. $J \mathrm{Am}$ Chem Soc 121: 2951-2955.

Thomas HC, Lamé MW, Wilson DW, Segall HJ 1996. Cell cycle alterations associated with covalent binding of monocrotaline pyrrole to pulmonary artery endothelial cell DNA. Toxicol Appl Pharm 141: 319-329.

Thomas HC, Lamé MW, Dunston SK, Segall HJ, Wilson DW 1998. Monocrotaline pyrrole induces apoptosis in pulmonary artery endothelial cells. Toxicol Appl Pharm 151: 236-244.

Thomson RG 1990. Patologia Veterinária Especial. São Paulo: Manole.

Tokarnia CH, Döbereiner J, Peixoto PV 2000. Plantas experimentalmente tóxicas para bovinos, porém incerto se de interesse pecuário. In: Tokarnia $\mathrm{CH}$, Döbereiner J, Peixoto PV (ed.) Plantas tóxicas do Brasil. 2. ed. Rio de Janeiro: Helianthus, p .240-242.

Wallace KB, Starkov AA 2000. Mitochondrial targets of drug toxicity. Annu Rev Pharmacol Toxicol 40: 353-388

Williams MC, Molyneux RJ 1987. Occurrence, concentration and toxicity of pyrrolizidine alkaloids in Crotalaria seeds. Weed Sci 35: 476-481.

Winter H, Seawright AA, Mattocks AR, Jukes R, Tshewang AR, Gurung BJ 1990. Pirrolizidine alkaloid poisoning in yaks. First report and confirmation by identification of sulphurbound pyrrolic metabolites of the alkaloids in preserved liver tissue. Aust Vet J 67: 411-412.

Yan CC, Huxtable RJ 1996. Effects of taurine and guanidioethane sulfnate on toxicity of the pyrrolizidine alkaloid monocrotaline. Biochem Pharmacol 51: 321-329. 